\title{
THE STRUCTURE AND STABILITY OF TWO-TEMPERATURE
}

\section{ACCRETION DISK}

\author{
MYEONG-GU PARK * \\ Department of Astronomy and Meteorology \\ Kyungpook National University \\ Taegu 702-701, KOREA
}

\begin{abstract}
The structure and stability of the two-temperature accretion disk around compact object with central soft-photon source is studied.
\end{abstract}

Key words: Accretion Disk, Black Holes, AGN, QSO

\section{Summary}

The structure and stability of the gas pressure dominated, thin accretion disk, cooled by Comptonization of the central soft photons is studied. Steady-state solutions have two branches: High-temperature (HT) solutions have very different ion and electron temperatures and correspond to the classic solutions of Shapiro, Lightman, and Eardley. Low-temperature (LT) solutions have same ion and electron temperature, which is very close to the Compton temperature of soft photons.

The linear analysis, allowing for the surface density perturbation and dynamics in the vertical direction, shows that LT disk is stable while HT disk is not. LT disk is stable because ions and electrons are locked to the Compton temperatre of the soft photons. HT disk generally has 4 local modes: (1) Heating mode grows in thermal time scale, $(5 / 3)(\alpha \omega)^{-1}$, where $\omega$ is Keplerian frequency. (2) Cooling mode decays in Compton time scale, $(2 / 5)\left(T_{e} / T_{i}\right)(\alpha \omega)^{-1} \ll(\alpha \omega)^{-1}$. (3) LightmanEardley mode decays in viscous time scale, $(8 / 11)\left(\Lambda / H_{0}\right)^{2}(\alpha \omega)^{-1}$, where $\Lambda$ is the wavelength of the perturbation and $H_{0}$ is the disk height. (4) Vertical oscillatory modes oscillate in Keplerian time scale, $(3 / 8)^{1 / 2} \omega^{-1}$ with growth rate $\propto\left(H_{0} / \Lambda\right)^{2}$. Including dynamics in the vertical direction does not change the stability behavior in general, adding only the oscillatory modes which gradually grow as $H_{0} / \Lambda$ increases.

Non-linear behaviour of the disk is followed by numerical integration. Cooling function covering both effectively optically thin and thick cases is used. Only the ion temperature perturbation is important and the disk either expands or collapses vertically, depending on the sign of the perturbation. When expanding, the ion temperature becomes very high while the electron temperature very low, resulting in runaway behavior due to the decreased Coulomb coupling, especially so if the ion velocity effect is considered. When collapsing, the disk approaches LT solutions and stabilizes at Compton temperature. 1.

* This work was supported by Korea Science and Engineering Foundation grant 923-0200-009-

T. J.-L. Courvoisier and A. Blecha: Multi-Wavelength Continuum Emission of AGN, 488.

(C) 1994 IAU. Printed in the Netherlands. 